\title{
Experimental Observation of Large Chern Numbers in Photonic Crystals
}

\author{
Scott A. Skirlo, ${ }^{1, *}$ Ling Lu, ${ }^{1, \dagger}$ Yuichi Igarashi, ${ }^{1,2}$ Qinghui Yan, ${ }^{1,3}$ John Joannopoulos, ${ }^{1}$ and Marin Soljačić ${ }^{1}$ \\ ${ }^{1}$ Department of Physics, Massachusetts Institute of Technology, Cambridge, Massachusetts 02139, USA \\ ${ }^{2}$ Smart Energy Research Laboratories, NEC Corporation, 34 Miyukigaoka, Tsukuba, Ibaraki 305-8501, Japan \\ ${ }^{3}$ The Electromagnetics Academy at Zhejiang University, Zhejiang University, Hangzhou 310027, China \\ (Received 15 April 2015; revised manuscript received 22 September 2015; published 14 December 2015)
}

Despite great interest in the quantum anomalous Hall phase and its analogs, all experimental studies in electronic and bosonic systems have been limited to a Chern number of one. Here, we perform microwave transmission measurements in the bulk and at the edge of ferrimagnetic photonic crystals. Band gaps with large Chern numbers of 2, 3, and 4 are present in the experimental results, which show excellent agreement with theory. We measure the mode profiles and Fourier transform them to produce dispersion relations of the edge modes, whose number and direction match our Chern number calculations.

DOI: 10.1103/PhysRevLett.115.253901

The Chern number [1] is an integer defining the topological phase in the quantum Hall effect (QHE) [2], which determines the number of topologically protected chiral edge modes. The quantum anomalous Hall effect (QAHE) possesses these same properties as an intrinsic property of the band structure with time reversal symmetry breaking [3,4]. Recent experiments have discovered the QAHE and its analogs in ferrimagnetic photonic crystals [5], magnetically doped thin films [6], and ultracold fermion lattices [7]. However, the Chern numbers observed in all of these systems were limited to \pm 1 . Finding larger Chern numbers would fundamentally expand the known topological phases [8-12].

Here, we provide the first explicit experimental observation of Chern numbers of magnitude 2, 3, and 4, by measuring bulk transmission, edge transmission, and the edge-mode dispersion relations in a ferrimagnetic photonic crystal. The excellent agreement between the experiment and the modeling allows us to identify various topological band gaps and map out the dispersion relations of one-way edge modes for the first time in any QHE or QAHE system in nature.

In a $2 \mathrm{D}$ system one can realize bands with nonzero Chern numbers, and generate the QAHE, by applying a $T$-breaking perturbation [13-15]. The Chern number is defined as the integral of the Berry flux over the entire Brillioun zone. When connected bands are gapped by a $T$-breaking perturbation, the bands will exchange equal and opposite Berry flux at each degenerate point, with the total Berry flux exchanged determining the Chern number. For instance, two isolated bands connected by one pair of Dirac points gapped by $T$-breaking will acquire $\pm 2 \pi$ Berry flux ( $\pi$ from each Dirac point) and a Chern number associated with the band gap ("gap Chern number") of \pm 1 . A general way to calculate the gap Chern number $\left(C_{\text {gap }}=\Sigma C_{i}\right)$ is to sum the Chern numbers of all the bands below the band gap [16]. A band gap with $C_{\text {gap }}=0$ is trivial, while a band gap with $C_{\text {gap }} \neq 0$ is topologically nontrivial.
PACS numbers: 42.79.Ta, 42.70.Qs, 42.79.Fm, 42.79.Gn

In our previous theoretical study, we found that the magnitude of the gap Chern number can be increased above one by simultaneously gapping multiple sets of Dirac and quadratic degeneracies. If Berry flux from the gapped degeneracies adds constructively, $C_{\text {gap }}$ can be large. In Fig. 1(a) we present a theoretical topological gap map for a 2D ferrimagnetic photonic crystal as a function of the externally applied magnetic field and the frequency, showing nontrivial band gaps with $C_{\text {gap }}$ from -4 to 3 . We studied this same square lattice in an experiment to verify these predictions.

The experimental configuration resembles a prior work which demonstrated $\left|C_{\text {gap }}\right|=1$ [5]. A square lattice of ferrimagnetic garnet rods is placed between two conductive copper plates. This configuration forms a parallel-plate waveguide, with the electric field perpendicular to the plate. Since the electric field for the fundamental mode is constant as a function of height, this is equivalent to a $2 \mathrm{D}$ system. The modes in the photonic crystal are excited by antennas attached to the top plate and fed to a network analyzer. Around the boundary of the system, we placed an absorber to minimize reflections and outside interference. We include an overhead image of one of the crystals we constructed in Fig. 1(d).

To observe the QAHE analog in the experiment, we break $T$ symmetry by applying a spatially uniform magnetic field to the ferrimagnetic garnet rods, which acquire off-diagonal imaginary parts in the permeability tensor [18]. Unlike electrons, the external magnetic field does not interact directly with photons. However, for this system, Maxwell's equations can be written in the exact same form as the Schrödinger equation with a periodic vector potential [15]. This makes the system an analog of the QAHE. Our photonic crystals were placed in the Massachusetts Institute of Technology (MIT) cyclotron magnet, and the magnetic field was swept between 0.03 and $0.55 \mathrm{~T}$ to characterize the transmission of the photonic crystal as a function of the magnetic field and the frequency. 

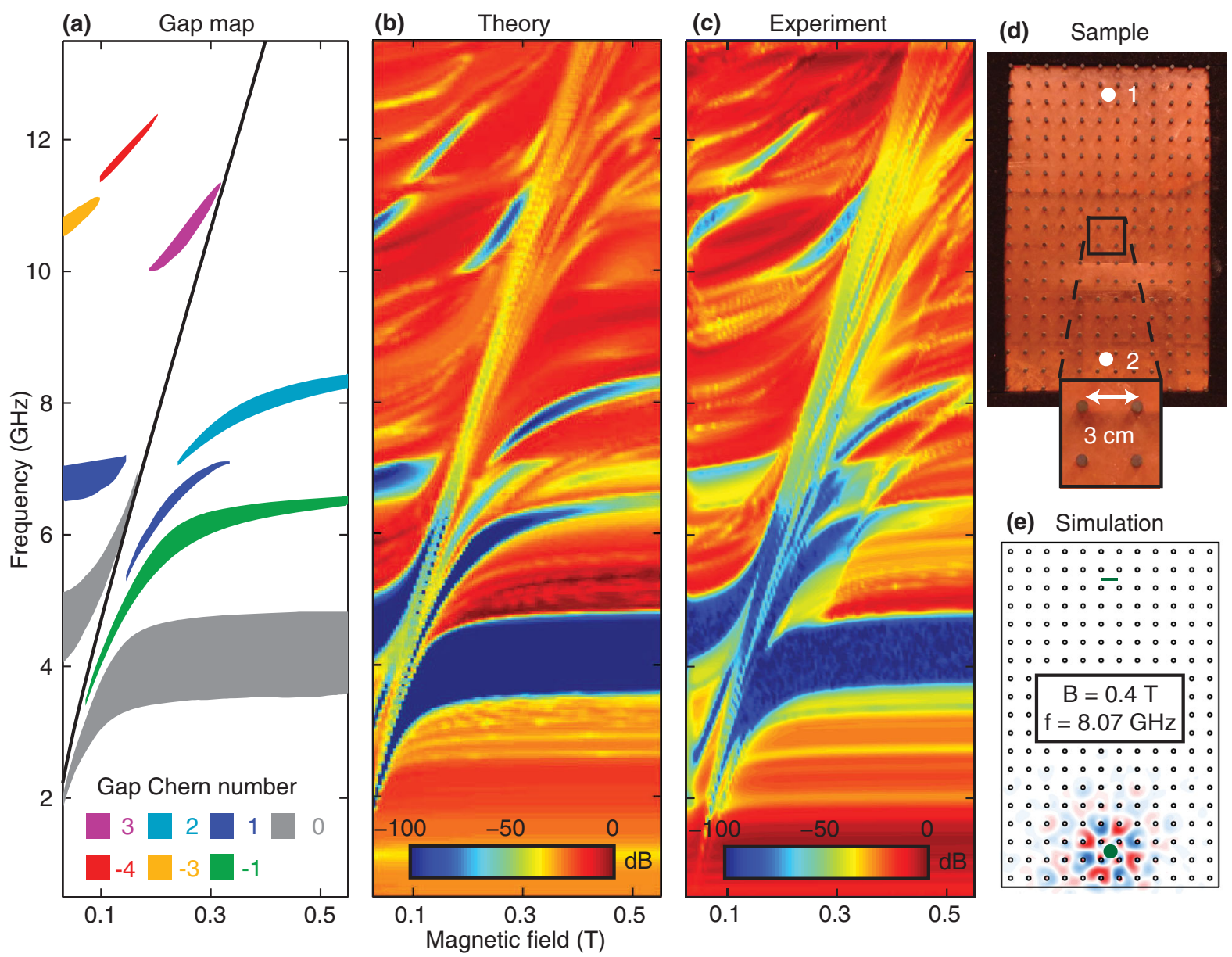

(e) Simulation

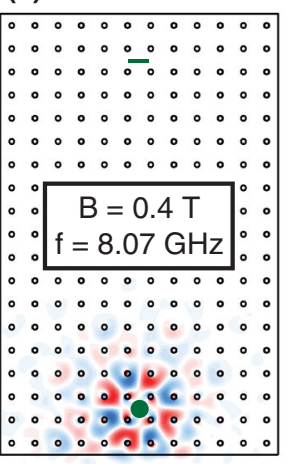

FIG. 1 (color online). Comparison of theoretical gap map and bulk transmission to experimental transmission measurement in a 2D ferrimagnetic photonic crystal. (a) Theoretical topological gap map as a function of the magnetic field and the frequency, with each band gap labeled by its gap Chern number. The diagonal black line indicates the resonance in the effective permeability (see the Supplemental Material [17]). (b) Theoretical bulk transmission. (c) Experimental bulk transmission. (d) Experimental configuration with the lattice geometry (top metal plate removed). The antenna locations are marked with 1 and 2. (e) Simulation geometry, with the green line representing the receiving antenna and the green circle representing the transmitting antenna.

We show the experimental transmission through a bulk photonic crystal in Fig. 1(c). Here, the color illustrates the amplitude of the transmission between the antennas in decibels $\left[S 12=20 \log \left(E_{\text {in }} / E_{\text {out }}\right)\right]$. In the plots there are several deep blue regions of low transmission that clearly correspond to the locations of band gaps in the gap map. The sweeping feature that extends diagonally across the figure is due to the gyromagnetic resonance of the ferrimagnetic garnet rods (see the Supplemental Material [17]). The resonant frequency of the effective permeability is plotted with a black line in Fig. 1(a).

In Fig. 1(b) we present the corresponding theoretical data for transmission through a lattice of the same size and dimension calculated with COMSOL. One of the transmission simulations is shown in Fig. 1(e). For direct comparison, the transmission data in Fig. 1(b) are plotted with the same color bar scale as the experiment in Fig. 1(c). The slight offset of about $0.04 \mathrm{~T}$ in the magnetic field between the theoretical and experimental plots is caused by demagnetization (see the Supplemental Material [17]). Clearly, the theoretical and experimental transmissions bear strong resemblance to each other and the topological gap map, showing that a square lattice of ferrimagnetic rods can contain a wide variety of different $C_{\text {gap }}$ numbers.

Several nontrivial band gaps in Fig. 1 and in the Supplemental Material [17] occur even at low magnetic fields. This indicates that topological effects can be achieved at low applied magnetic fields $(\sim 0.03 \mathrm{~T})$ enabling various studies and applications. Furthermore, these same band gaps would remain open at zero external magnetic field by using ferrimagnetic materials with remanent magnetization [19]; this way, a future experiment could be performed even without external magnetic fields.

One-way edge modes are present at the boundary between two crystals with a nontrivial and a trivial band gap, respectively, or at the boundary between crystals with 
nontrivial band gaps with different $C_{\text {gap }}[4,20]$. If the band gaps of two neighboring crystals overlap in frequency, the number of edge states in the shared frequency gap is determined by the difference between the gap Chern numbers of each crystal. The sign of this difference determines the directions of the edge states. This means that with the nontrivial band gaps we found, constructing one-way waveguides with up to seven modes is possible. If one of the materials is trivial $\left(C_{\text {gap }}=0\right)$, like metal or air, the number of edge states equals the gap Chern number of the crystal, with the sign of this number determining their directions.

To provide more evidence of the topological state of these band gaps and the one-way modes, we modified the setup to include a highly conductive copper boundary at the edge of the crystal. This boundary acts as a mirror with a trivial band gap. We place two antennas near this edge on each side of the sample and measure the transmission between them. In Figs. 2(a) and 2(b) we present both the $S 12$ and $S 21$ parameters to describe the directiondependent transmission of the edge modes along the metal boundary. $S 12$ refers to exciting the second antenna and measuring with the first antenna, while $S 21$ is the opposite.

The band gaps that are nontrivial $\left(C_{\text {gap }} \neq 0\right)$ can be identified in Fig. 2 because they will have directiondependent edge transmission. Specifically, the nontrivial band gaps measured in Fig. 1(c) will appear in either Fig. 2(a) or Fig. 2(b), but not both. We show this explicitly for the $C_{\text {gap }}=-4$ band gap by highlighting the directiondependent transmission with gray boxes. This arises from the directional edge states as follows. In one direction, the group velocity of the edge modes is opposite that required (a)

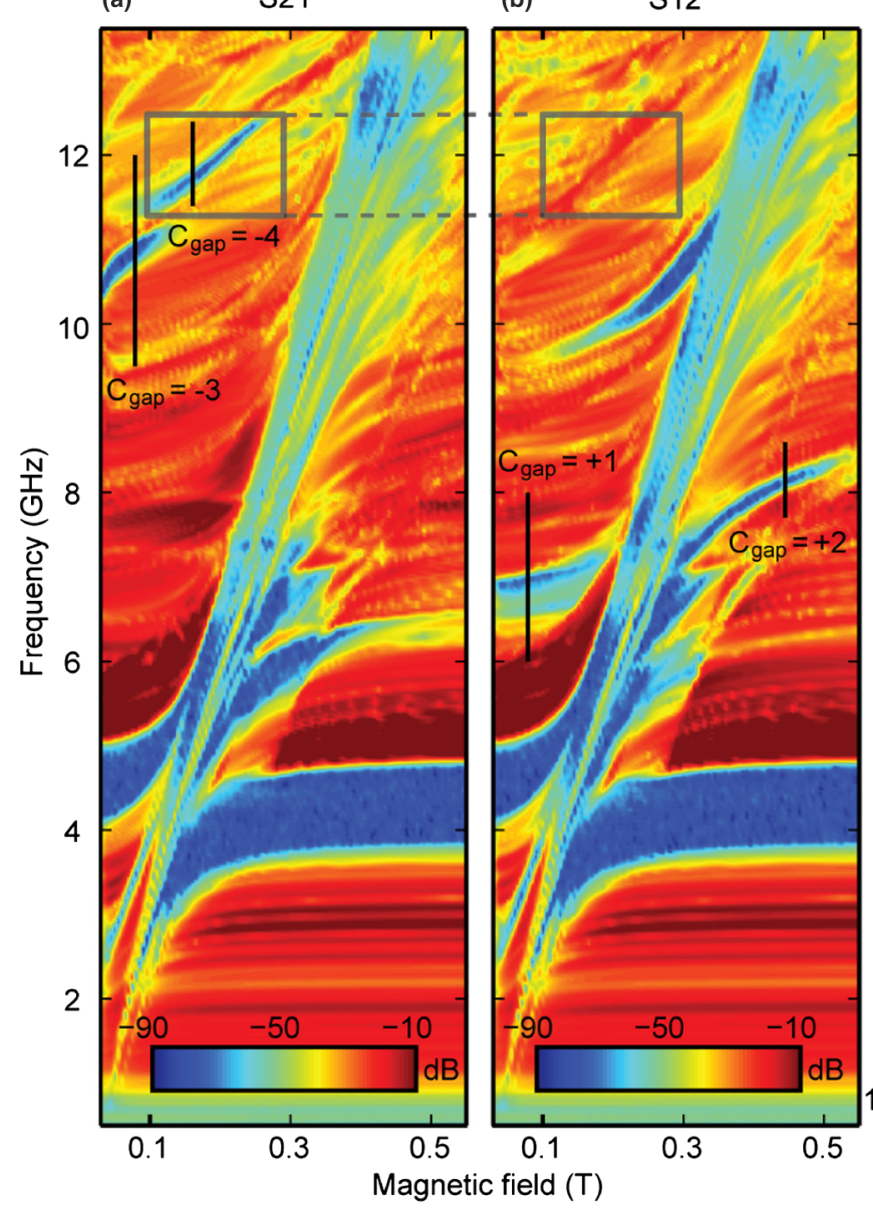

(c) FT of mode profile
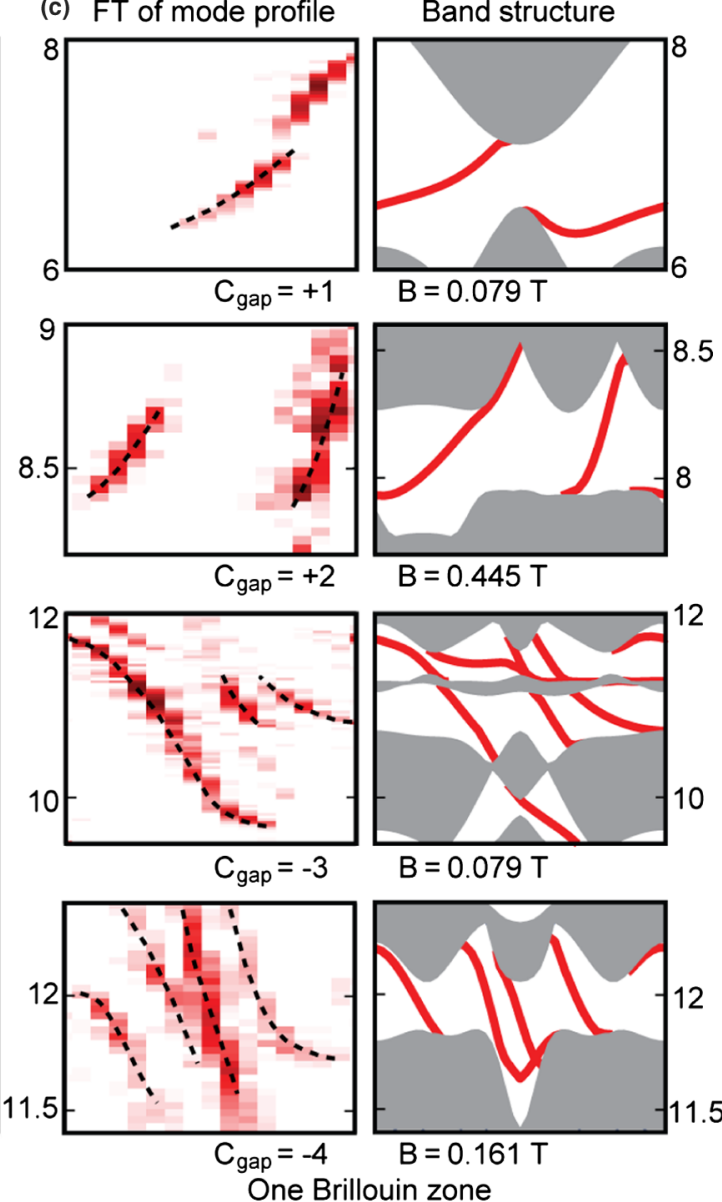

One Brillouin zone

FIG. 2 (color online). Experimental edge transmission measurement and Fourier transform (FT) of mode profiles along the copper boundary. (a) $S 21$. (b) $S 12$. The band gaps that are nontrivial have direction-dependent edge transmission because the interface of a nontrivial band gap with a trivial band gap (copper boundary) supports one-way modes. In (a) and (b) this causes the nontrivial bulk band gaps from Fig. 1(c) to be present in one direction (e.g., S12) and absent in the other (e.g., S21), which we highlight for the $C_{\text {gap }}=-4$ band gap with black boxes. The trivial band gaps around $4 \mathrm{GHz}$ do not support one-way modes, and so they do not exhibit direction-dependent transmission. (c) Experimental FT of edge-mode profiles and the theoretical edge band structures with the edge modes in red and the bulk bands in gray. The range of wave vectors included in both plots is the same and includes only one Brillouin zone. The number of one-way edge modes in both sets of panels agrees with $\left|C_{\text {gap }}\right|$ from Fig. 1(a), while the sign of $C_{\text {gap }}$ is consistent with the theoretical group velocity (from the edge-mode dispersion) and the directional transmission in (a) and (b). 
to travel to the receiving antenna, so the transmission measurement will record the bulk band gap. However, in the other direction, the group velocity of the edge modes is in the same direction as is required to get to the receiving antenna, so the band gap will appear to be nonexistant. Trivial band gaps $\left(C_{\text {gap }}=0\right)$ around $4 \mathrm{GHz}$ do not support one-way edge modes and thus do not exhibit directiondependent transmission at the edge.

To further study the topological nature of these band gaps, we measured the mode profile at the edge of the photonic crystal. We accomplished this by mounting one antenna for excitation to the lower plate, and another small dipole antenna for measurement to the upper plate [21]. During the measurement, the upper plate was translated a total of $47 \mathrm{~cm}$ in $2.5 \mathrm{~mm}$ steps. At each step both the phase and the amplitude of the electric field was recorded (see the Supplemental Material [17]). From this spatial data, the mode profile in the waveguide can be reconstructed. The Fourier transform of the mode profile produces the dispersion relation of the waveguide, which we present on the left-hand side of Fig. 2(c).

In Fig. 2(c) on the right-hand side, we include a comparison with the edge band calculations, with the bulk bands in gray and the edge modes in red. It is clear that the calculated edge-mode dispersion shows an excellent agreement with the dispersion relations extracted from experiments. The number of edge modes is equal to the gap Chern number for each inset. The sign of $C_{\text {gap }}$ is consistent with the group velocity of the edge modes and agrees with the directional edge transmission data in Figs. 2(a) and 2(b). In the Supplemental Material [17] we present additional simulations validating these results for $C_{\text {gap }}=-3$ and -4 . These results constitute the first direct measurement of one-way edge-mode dispersion in any QHE system.

To further study the gap Chern numbers of the observed topological band gaps, we construct a topological oneway circuit [11]. As illustrated in Fig. 3(d), this consists of a $C_{\text {gap }}=2(a=3.0 \mathrm{~cm})$ crystal and a $C_{\text {gap }}=1$ $(a=2.4 \mathrm{~cm})$ crystal, with a copper boundary on the edge. We present the design and calculations characterizing the $C_{\text {gap }}=1$ crystal in the Supplemental Material [17], while the results from the $C_{\text {gap }}=2$ crystal are shown in Figs. 1 and 2. From the rules described earlier, there will be two edge states flowing downwards between the metal boundary and the $C_{\text {gap }}=2$ crystal, as indicated with arrows in Fig. 3(d). These edge states will "split" at the junction, with one edge state flowing away along the boundary between the $C_{\text {gap }}=1$ and the $C_{\text {gap }}=2$ crystal and the other continuing along the metal and $C_{\text {gap }}=1$ crystal interface.

In Figs. 3(a)-3(c) we present the transmission between ports 1-3 and a fourth port located at the junction, as labeled in Fig. 3(d). The highlighted yellow region indicates the shared band gap between the $C_{\text {gap }}=1$ crystal and the $C_{\text {gap }}=2$ crystal. For each of the measurements, it is clear

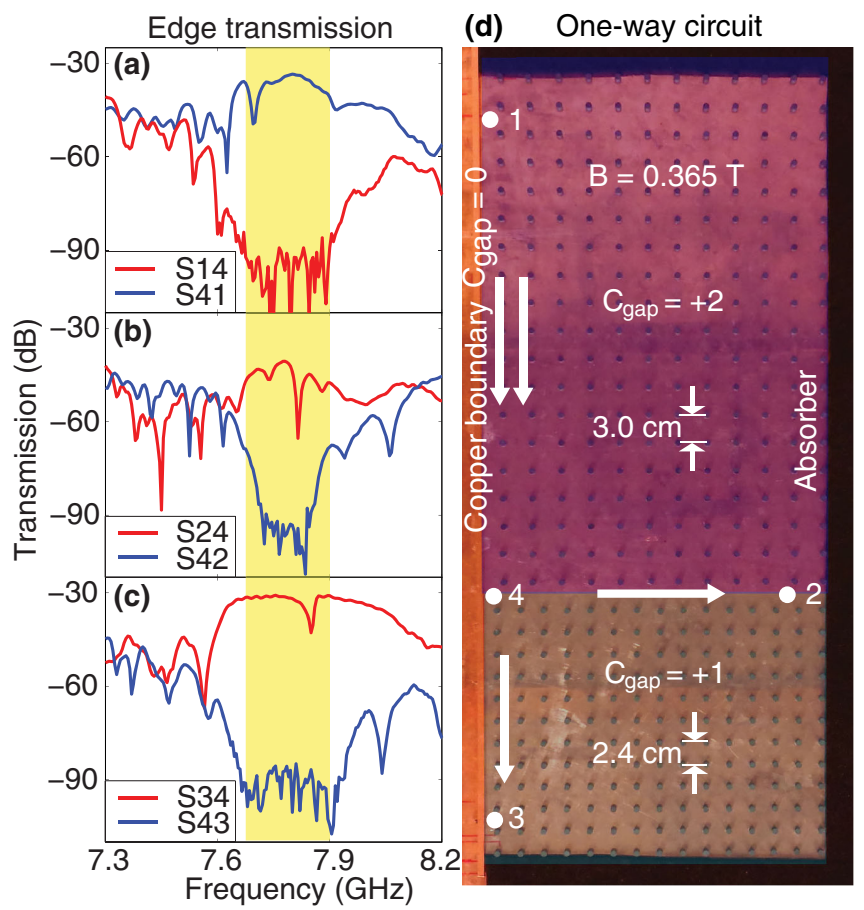

FIG. 3 (color online). Topological one-way circuit implemented using $C_{\text {gap }}=1(a=2.4 \mathrm{~cm})$ and $C_{\text {gap }}=2(a=3.0 \mathrm{~cm})$ photonic crystals. (a)-(c) Transmission plots showing edge transmission between antennas at 1,2, and 3, and antenna 4 located at the center. Shared bulk band gap for $C_{\text {gap }}=1$ and $C_{\text {gap }}=2$ crystals is highlighted in yellow. (d) Experimental configuration illustrating crystals with copper boundary $\left(C_{\text {gap }}=0\right)$ on the left and antenna locations 1-4. Arrows indicate the theoretical direction and the number of the edge states at each interface. The transmission data is consistent with predicted edge state directions, which confirms that the upper crystal has $C_{\text {gap }}>1$.

that in one direction we have a strong band gap, with a signal level at the noise floor of about $-100 \mathrm{~dB}$, while in the opposite direction there is 50 to $60 \mathrm{~dB}$ more of a transmission. These edge state directions are consistent with the theoretical predictions and prove the existence of $C_{\text {gap }}>1$ for the upper crystal. The results from Fig. 3 were obtained under an applied magnetic field of $0.365 \mathrm{~T}$, although there was a window extending from approximately 0.32 to $0.4 \mathrm{~T}$, where the $C_{\text {gap }}=2$ and $C_{\text {gap }}=1$ band gaps from each crystal were well aligned (see the Supplemental Material [17]).

In conclusion, we experimentally constructed a square lattice ferrimagnetic photonic crystal with a band structure comprising high $C_{\text {gap }}$ ( -4 to 3 ) band gaps and measured the dispersion relations of the multimode one-way edge waveguides. Fundamentally, having band gaps with higher gap Chern numbers greatly expands the phases available for topological photonics. These results can potentially enable multimode one-way waveguides with high capacity and coupling efficiencies, as well as many other devices [22-25]. A topological photonic circuit can also be made 
by interfacing photonic crystals with various $C_{\text {gap }}$ 's, with one-way edge states combining together or splitting off at the junctions. Given the rapidly expanding literature on the QAHE and its analogs for $\left|C_{\text {gap }}\right|=1$ [4,26-31], many more avenues of research are now possible because of the greater range of topological phases that can be investigated. Our approach can be readily extended to other systems of bosonic particles such as magnons [32], excitons [33], and phonons $[34,35]$.

We acknowledge Carl E. Patton, Liang Fu, Bo Zhen, Ido Kaminer, Yichen Shen, Zheng Weng, and Hongsheng Chen for the discussions, Ulrich Becker and Peter Fisher for their assistance with the MIT cyclotron magnet, and Xiangdong Liang for the numerical assistance. S. S. was supported by the MIT Tom Frank Fellowship and the NSF Fellowship. L. L. was supported in part by the MRSEC Program of the NSF under Grant No. DMR-1419807. Q. Y. was supported by the National Natural Science Foundation of China under Grant No. 61322501. The fabrication part of the effort was paid for by the MIT S3TEC EFRC of the U.S. DOE under Grant No. DE-SC0001299. This work was also supported in part by ARO through ISN, Grant No. W911NF-13D-0001.

*sskirlo@mit.edu

†linglu@mit.edu

[1] D. J. Thouless, M. Kohmoto, M. P. Nightingale, and M. den Nijs, Quantized Hall Conductance in a Two-Dimensional Periodic Potential, Phys. Rev. Lett. 49, 405 (1982).

[2] K. v. Klitzing, G. Dorda, and M. Pepper, New Method for High-Accuracy Determination of the Fine-Structure Constant Based on Quantized Hall Resistance, Phys. Rev. Lett. 45, 494 (1980).

[3] F. D. M. Haldane, Model for a Quantum Hall Effect without Landau Levels: Condensed-Matter Realization of the "Parity Anomaly", Phys. Rev. Lett. 61, 2015 (1988).

[4] L. Lu, J. D. Joannopoulos, and M. Soljačić, Topological photonics, Nat. Photonics 8, 821 (2014).

[5] Z. Wang, Y. Chong, J. D. Joannopoulos, and M. Soljačić, Observation of unidirectional backscattering-immune topological electromagnetic states, Nature (London) 461, 772 (2009).

[6] C. Chang et al., Experimental observation of the quantum anomalous Hall effect in a magnetic topological insulator, Science 340, 167 (2013).

[7] G. Jotzu, M. Messer, R. Desbuquois, M. Lebrat, T. Uehlinger, D. Greif, and T. Esslinger, Experimental realization of the topological Haldane model with ultracold fermions, Nature (London) 515, 237 (2014).

[8] H. Jiang, Z. H. Qiao, W. Liu, and Q. Niu, Quantum anomalous Hall effect with tunable Chern number in magnetic topological insulator film, Phys. Rev. B 85, 045445 (2012).

[9] J. Wang, B. Lian, H. Zhang, Y. Xu, and S. Zhang, Quantum Anomalous Hall Effect with Higher Plateaus, Phys. Rev. Lett. 111, 136801 (2013).
[10] C. Fang, M. J. Gilbert, and B. A. Bernevig, Large-ChernNumber Quantum Anomalous Hall Effect in Thin-Film Topological Crystalline Insulators, Phys. Rev. Lett. 112, 046801 (2014).

[11] S. A. Skirlo, L. Lu, and M. Soljačić, Multimode One-Way Waveguides of Large Chern Numbers, Phys. Rev. Lett. 113, 113904 (2014).

[12] P. M. Perez-Piskunow, L. E. F. Foa Torres, and G. Usaj, Hierarchy of Floquet gaps and edge states for driven honeycomb lattices, Phys. Rev. A 91, 043625 (2015).

[13] F. D. M. Haldane and S. Raghu, Possible Realization of Directional Optical Waveguides in Photonic Crystals with Broken Time-Reversal Symmetry, Phys. Rev. Lett. 100, 013904 (2008).

[14] S. Raghu and F. D. M. Haldane, Analogs of quantum-Halleffect edge states in photonic crystals, Phys. Rev. A 78, 033834 (2008).

[15] Z. Wang, Y. D. Chong, J. D. Joannopoulos, and M. Soljačić, Reflection-Free One-Way Edge Modes in a Gyromagnetic Photonic Crystal, Phys. Rev. Lett. 100, 013905 (2008).

[16] T. Fukui, Y. Hatsugai, and H. Suzuki, Chern numbers in discretized Brillouin zone: Efficient method of computing (spin) Hall conductances, J. Phys. Soc. Jpn. 74, 1674 (2005).

[17] See Supplemental Material at http://link.aps.org/ supplemental/10.1103/PhysRevLett.115.253901 for a description of the experimental methods, the ferrimagnetic material model, additional mode profile measurements, additional results for the $C_{\text {gap }}=1$ crystal, and finally further details of the Chern number calculations.

[18] D. M. Pozar, Microwave Engineering, 2nd ed. (Wiley, New York, 1998).

[19] J. J. Green and F. Sandy, Microwave characterization of partially magnetized ferrites, IEEE Trans. Microwave Theory Tech. 22, 641 (1974).

[20] Y. Hatsugai, Chern Number and Edge States in the Integer Quantum Hall Effect, Phys. Rev. Lett. 71, 3697 (1993).

[21] S. Li et al., Probing electric field in an enclosed field mapper for characterizing metamaterials, Int. J. Antennas Propag. 2014, 728756 (2014).

[22] C. He, X.-L. Chen, M.-H. Lu, X.-F. Li, W.-W. Wan, X.-S. Qian, R.-C. Yin, and Y.-F. Chen, Tunable one-way crosswaveguide splitter based on gyromagnetic photonic crystal, Appl. Phys. Lett. 96, 111111 (2010).

[23] Y. Yang, Y. Poo, R. Wu, Y. Gu, and P. Chen, Experimental demonstration of one-way slow wave in waveguide involving gyromagnetic photonic crystals, Appl. Phys. Lett. 102, 231113 (2013).

[24] Y. Poo, R. Wu, Z. Lin, Y. Yang, and C. T. Chan, Experimental Realization of Self-Guiding Unidirectional Electromagnetic Edge States, Phys. Rev. Lett. 106, 093903 (2011).

[25] J. Fu, J. Lian, R. Liu, L. Gan, and Z. Li, Unidirectional channel-drop filter by one-way gyromagnetic photonic crystal waveguides, Appl. Phys. Lett. 98, 211104 (2011).

[26] M. Hafezi, E. A. Demler, M. D. Lukin, and J. M. Taylor, Robust optical delay lines with topological protection, Nat. Phys. 7, 907 (2011).

[27] K. Fang, Z. Yu, and S. Fan, Realizing effective magnetic field for photons by controlling the phase of dynamic modulation, Nat. Photonics 6, 782 (2012). 
[28] A. B. Khanikaev, S. Hossein Mousavi, W.-K. Tse, M. Kargarian, A. H. MacDonald, and G. Shvets, Photonic topological insulators, Nat. Mater. 12, 233 (2013).

[29] Y.E. Kraus, Y. Lahini, Z. Ringel, M. Verbin, and O. Zilberberg, Topological States and Adiabatic Pumping in Quasicrystals, Phys. Rev. Lett. 109, 106402 (2012).

[30] M. C. Rechtsman, J. M. Zeuner, Y. Plotnik, Y. Lumer, D. Podolsky, F. Dreisow, S. Nolte, M. Segev, and A. Szameit, Photonic Floquet topological insulators, Nature (London) 496, 196 (2013).

[31] W. Chen et al., Experimental realization of photonic topological insulator in a uniaxial metacrystal waveguide, Nat. Commun. 5, 196 (2014).
[32] A. Mook, J. Henk, and I. Mertig, Edge states in topological magnon insulators, Phys. Rev. B 90, 024412 (2014).

[33] J. Yuen-Zhou, S. K. Saikin, N. Y. Yao, and A. AspuruGuzik, Topologically protected excitons in porphyrin thin films, Nat. Mater. 13, 1026 (2014).

[34] Z. Yang, F. Gao, X. Shi, X. Lin, Z. Gao, Y. Chong, and B. Zhang, Topological Acoustics, Phys. Rev. Lett. 114, 114301 (2015).

[35] P. Wang, L. Lu, and K. Bertoldi, Topological Phononic Crystals with One-Way Elastic Edge Waves, Phys. Rev. Lett. 115, 104302 (2015). 